\title{
FLOCKING WITH SHORT-RANGE INTERACTIONS
}

\author{
JAVIER MORALES, JAN PESZEK, AND EITAN TADMOR
}

\begin{abstract}
We study the large-time behavior of continuum alignment dynamics based on Cucker-Smale (CS)-type interactions which involve short-range kernels, that is, communication kernels with support much smaller than the diameter of the crowd. We show that if the amplitude of the interactions is larger than a finite threshold, then unconditional hydrodynamic flocking follows. Since we do not impose any regularity nor do we require the kernels to be bounded, the result covers both regular and singular interaction kernels.

Moreover, we treat initial densities in the general class of compactly supported measures which are required to have positive mass on average (over balls at small enough scale), but otherwise vacuum is allowed at smaller scales. Consequently, our arguments of hydrodynamic flocking apply, mutatis mutandis, to the agent-based CS model with finitely many Dirac masses. In particular, discrete flocking threshold is shown to depend on the number of dense clusters of communication but otherwise does not grow with the number of agents.
\end{abstract}

\section{Contents}

1. Introduction and statement of main results 1

2. Staying uniformly away from vacuum 6

3. Chain connectivity 6

4. Proof of the main results 8

5. Uniformly bounded density away from vacuum 13

6. Closing remarks 14

References 14

\section{IntRoduCtion AND STATEMENT OF MAIN RESULTS}

Our starting point is the celebrated Cucker-Smale (CS) agent-based model [4, 5] which governs the alignment dynamics of $N$ agents - each identified by its (position, velocity) pair, $\left(\mathbf{x}_{i}(t), \mathbf{v}_{i}(t)\right) \in \Omega \times \mathbb{R}^{d}$,

$$
\left\{\begin{array}{l}
\dot{\mathbf{x}}_{i}=\mathbf{v}_{i} \\
\dot{\mathbf{v}}_{i}=\frac{\kappa}{N} \sum_{j=1}^{N} \phi\left(\left|\mathbf{x}_{i}-\mathbf{x}_{j}\right|\right)\left(\mathbf{v}_{j}-\mathbf{v}_{i}\right),
\end{array}\right.
$$

and is subject to prescribed initial conditions $\left(\mathbf{x}_{i}(0), \mathbf{v}_{i}(0)\right)=\left(\mathbf{x}_{i 0}, \mathbf{v}_{i 0}\right)$. Here we study the $d$-dimensional ambient space where $\Omega$ is either $\mathbb{T}^{d}$ or $\mathbb{R}^{d}$.

Date: May 2, 2019.

1991 Mathematics Subject Classification. 92D25, 35Q35, 76N10.

Key words and phrases. Alignment, Cucker-Smale, agent-based system, large-crowd hydrodynamics, interaction kernels, short-range, chain connectivity, flocking.

Acknowledgment. Research was supported by NSF grants DMS16-13911, RNMS11-07444 (KI-Net) and ONR grant N00014-1812465. JP was also supported by the Polish MNiSW grant Mobilność Plus no. $1617 / \mathrm{MOB} / \mathrm{V} / 2017 / 0$. 
The large crowd dynamics, $N \rightarrow \infty$, is captured by the hydrodynamic description

$$
\left\{\begin{array}{l}
\partial_{t} \rho+\nabla_{\mathbf{x}} \cdot(\rho \mathbf{u})=0 \\
\partial_{t} \mathbf{u}+\mathbf{u} \cdot \nabla_{\mathbf{x}} \mathbf{u}=\kappa \int_{\Omega} \phi(|\mathbf{x}-\mathbf{y}|)(\mathbf{u}(\mathbf{y})-\mathbf{u}(\mathbf{x})) \mathrm{d} \rho(\mathbf{y}),
\end{array}\right.
$$

which governs the density and velocity of the crowd, $(\rho(t, \mathbf{x}), \mathbf{u}(t, \mathbf{x}))$ at $(t, \mathbf{x}) \in\left(\mathbb{R}_{+} \times \Omega\right)$, subject to prescribed data $(\rho(0, \mathbf{x}), \mathbf{u}(0, \mathbf{x}))=\left(\rho_{0}(\mathbf{x}), \mathbf{u}_{0}(\mathbf{x})\right)$.

The main feature of both - the agent-based model (1.1) and its large crowd hydrodynamic description (1.2), is the communication or interaction kernel, $\phi:[0, \infty) \rightarrow[0, \infty)$, which controls pairwise interactions depending on the geometric distances of agents at $(t, \mathbf{x})$ and $(t, \mathbf{y})$. Here $\kappa>0$ quantifies the amplitude of the interaction and we distinguish between two main classes of interactions, involving long-range and short-range kernels.

1.1. Long-range interactions. The role of $\phi$ in determining the large-time behavior becomes evident once we consider the energy fluctuations associated with (1.1) or (1.2). Let $\mathcal{S}(t)$ denote the support of $\rho(t, \cdot)$ and assume, without loss of generality, that $\rho_{0}$ has a unit mass, then conservation of mass implies

$$
\int_{\mathcal{S}(t)} \mathrm{d} \rho(t, \cdot) \equiv 1, \quad \mathcal{S}(t):=\operatorname{supp} \rho(t, \cdot) .
$$

The basic bookkeeping associated with (1.2) quantifies the decay rate of the $L^{2}$-energy fluctuations $^{1}$

$$
|\delta \mathbf{u}(t)|_{2}^{2}:=\int_{\mathcal{S}(t) \times \mathcal{S}(t)}|\mathbf{u}(t, \mathbf{x})-\mathbf{u}(t, \mathbf{y})|^{2} \mathrm{~d} \rho(\mathbf{x}) \mathrm{d} \rho(\mathbf{y}),
$$

in terms of the enstrophy,

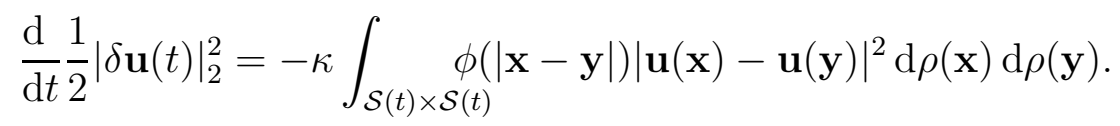

The enstrophy on the right admits the obvious lower-bound

$$
\int_{\mathcal{S}(t) \times \mathcal{S}(t)} \phi(|\mathbf{x}-\mathbf{y}|)|\mathbf{u}(\mathbf{x})-\mathbf{u}(\mathbf{y})|^{2} \mathrm{~d} \rho(\mathbf{x}) \mathrm{d} \rho(\mathbf{y}) \geqslant \min _{h \leqslant \operatorname{diam}(\mathcal{S}(t))} \phi(h) \times|\delta \mathbf{u}(t)|_{2}^{2} .
$$

Since the diameter of velocity field, $\sup _{\mathbf{x}, \mathbf{y} \in \mathcal{S}(t)}|\mathbf{u}(t, \mathbf{x})-\mathbf{u}(t, \mathbf{y})|$, remains bounded, the support of $\rho$ cannot expand faster than

$$
\operatorname{diam}(\mathcal{S}(t)) \leqslant \operatorname{diam}\left(\mathcal{S}_{0}\right)+\left|\delta \mathbf{u}_{0}\right|_{\infty} \cdot t, \quad|\delta \mathbf{u}(t)|_{\infty}:=\sup _{\mathbf{x}, \mathbf{y} \in \mathcal{S}(t)}|\mathbf{u}(t, \mathbf{x})-\mathbf{u}(t, \mathbf{y})|,
$$

and hence (1.4) implies the unconditional flocking behavior of (1.2), in the sense that

$$
\int_{0}^{\infty} \phi(h) \mathrm{d} h=\infty \leadsto|\delta \mathbf{u}(t)|_{2}^{2} \stackrel{t \rightarrow \infty}{\longrightarrow} 0,
$$

holds for all initial data which give rise to smooth solutions. Thus, if $\phi$ is a long-range interaction kernel satisfying the 'fat tail' condition (1.5), then 'smooth solutions must flock', [27], and the existence of smooth solutions is known in $\Omega=\mathbb{T}^{1}$, [7, 23, 24, 25], in $\Omega=\mathbb{R}^{2}$ [14], and with small data in $\Omega=\mathbb{T}^{d}$ and $\Omega=\mathbb{R}^{d}[22,6]$.

\footnotetext{
${ }^{1}$ Here and below we use $\delta$. to denote fluctuations, $\delta W \equiv(\delta W)(\mathbf{x}, \mathbf{y}):=W(\mathbf{x})-W(\mathbf{y})$ with the corresponding weighted norms taken on the product space $\mathcal{S} \times \mathcal{S}$, e.g., $|\delta \mathbf{u}|_{2}^{2}=\int|\mathbf{u}(\mathbf{x})-\mathbf{u}(\mathbf{y})|^{2} \mathrm{~d} \rho(\mathbf{x}) \mathrm{d} \rho(\mathbf{y})$. Likewise, $(\delta \mathbf{v})_{i j}=\mathbf{v}_{i}-\mathbf{v}_{j}$ with $|\delta \mathbf{v}|_{\infty}=\max _{i, j}\left|\mathbf{v}_{i}-\mathbf{v}_{j}\right|$ etc.
} 
The situation is completely analogous to the agent-based case supported on the discrete set $\mathcal{S}(t):=\left\{\mathbf{x}_{i}(t)\right\}_{i=1}^{N}$, where the decay of energy fluctuations is controlled by

$$
\frac{\mathrm{d}}{\mathrm{d} t}|\delta \mathbf{v}(t)|_{2}^{2} \leqslant-\kappa \min _{h \leqslant \operatorname{diam}(\mathcal{S}(t))} \phi(h) \times|\delta \mathbf{v}(t)|_{2}^{2}, \quad|\delta \mathbf{v}(t)|_{2}^{2}:=\frac{1}{N^{2}} \sum_{i, j=1}^{N}\left|\mathbf{v}_{i}(t)-\mathbf{v}_{j}(t)\right|^{2} .
$$

But since the diameter of velocities, $\max _{1 \leqslant i, j \leqslant N}\left|\mathbf{v}_{i}(t)-\mathbf{v}_{j}(t)\right|$, remains bounded, the diameter of positions grows at most linearly in time

$$
\operatorname{diam}(\mathcal{S}(t)) \leqslant \operatorname{diam}\left(\mathcal{S}_{0}\right)+|\delta \mathbf{v}(0)|_{\infty} \cdot t, \quad|\delta \mathbf{v}(t)|_{\infty}:=\max _{1 \leqslant i, j \leqslant N}\left|\mathbf{v}_{i}(t)-\mathbf{v}_{j}(t)\right|,
$$

and we conclude that long-range communication kernels with fat-tail imply flocking, [12, 11, $17]$

$$
\int^{\infty} \phi(h) \mathrm{d} h=\infty \leadsto|\delta \mathbf{v}(t)|_{2}^{2} \stackrel{t \rightarrow \infty}{\longrightarrow} 0 .
$$

1.2. Short-range interactions. From the perspective of practical applications, it is important to understand the large-time behavior of alignment dynamics driven by short range kernels, specifically — when $\operatorname{diam}(\operatorname{supp} \phi)$ is strictly less than the support of the crowd, $\operatorname{diam}(\mathcal{S}(t))$. The fascinating and non-trivial aspect is to understand when and how such short-range interactions lead to the emergence of large-scale patterns, which in our case are realized by the flocking behavior approaching the average ${ }^{2} \overline{\mathbf{u}}_{0}$,

$$
\int_{\Omega}\left|\mathbf{u}(t, \mathbf{x})-\overline{\mathbf{u}}_{0}\right|^{2} \mathrm{~d} \rho(\mathbf{x}) \longrightarrow 0, \quad \overline{\mathbf{u}}_{0}:=\int_{\mathcal{S}_{0}} \mathbf{u}_{0}(\mathbf{x}) \mathrm{d} \rho(\mathbf{x}),
$$

and likewise $\left|\mathbf{v}_{i}(t)-\overline{\mathbf{v}}_{0}\right| \rightarrow 0$ in the discrete case. The dynamics of such short-range interactions lies outside the 'fat-tale' regime (1.5),(1.7) and is the main focus of the present work.

We begin by fixing an essential support of $\phi$ of size $R$ where it is assumed to be bounded away from zero,

$$
\phi:(0, \infty) \rightarrow(0, \infty), \quad \phi(h) \chi_{[0, R]}(h) \geqslant 1>0 \text { for } h \in[0, R] .
$$

We emphasize that the only requirement made in (1.8) is that the kernel has a finite-range, $R$, of non-negligible interaction which is scaled here as $\phi(h) \geqslant 1$, but otherwise, we make no reference to the behavior of $\phi$ outside $[0, R]$. In particular, a smooth $\phi$ may have a compact support, $\operatorname{say}, \operatorname{supp}(\phi)=[0,2 R]$, or - since we do not impose any regularity restrictions, $\phi$ can be cut-off at $h=R$. In either case, $\phi$ is not restricted to maintain a direct communication between every two agents in the crowd. We summarize by introducing the main spatial scales involved in the problem, namely

(1.9a) The diameter of the initial 'crowd' distribution: $D_{\mathcal{S}_{0}}:=\sup _{\mathbf{x}, \mathbf{y} \in \mathcal{S}_{0}}|\mathbf{x}-\mathbf{y}|$,

(1.9b) The essential diameter of the communication : $\quad D_{\phi}:=\sup \left\{r \mid \min _{h \leqslant r} \phi(h) \geqslant 1\right\}$.

We specifically address the case of short-range interactions $D_{\phi} \ll D_{\mathcal{S}_{0}}$. Moreover, since we do not impose any boundedness of $\phi,(1.8)$ includes both - bounded communication kernels, $[4,5,12,11,2,17]$, and singular ones [19, 20, 21, 23, 24, 25, 7].

\footnotetext{
${ }^{2}$ Recall that $\rho$ is a probability measure
} 
The flocking behavior in such cases hinges on the connectivity of the communication throughout the crowd: in the discrete case, it is the graph connectivity of the adjacency matrix $\left\{\phi\left(\left|\mathbf{x}_{i}(t)-\mathbf{x}_{j}(t)\right|\right)\right\},[17$, theorem 4.2]. Here we show that in the continuum, it is the chain connectivity at a communication scale that matters (see assumption 3.2 below): if chain connectivity at the communication scale persists in time then the dynamics will converge to a flock. Our main results show that if the amplitude of alignment is strong enough, $\kappa>\kappa_{0}$, such chain connectivity persists and hence uniform flocking follows.

Theorem 1.1 (Emergence of hydrodynamic flocking). Let $(\rho, \mathbf{u})$ be a strong solution of $(1.2),(1.8)$ in either $\mathbb{T}^{d}$ or $\mathbb{R}^{d}$ subject to compactly supported initial data $\left(\rho_{0}, \mathbf{u}_{0}\right) \in$ $\left(L^{1}\left(\mathcal{S}_{0}\right), C^{1}\left(\mathcal{S}_{0}\right)\right)$. Assume $\rho_{0}$ is normalized to have a unit mass and it satisfies the following (see assumption 3.2 below):

(i) Its support is chain connected with $n(r)$ balls at scale $r$ for some $r \leqslant \frac{1}{6} D_{\phi}($ see (3.2));

(ii) It has a minimal average mass $m>0$ at scale $\frac{r}{100}$ (see (3.3)).

Then, there exist constants $\eta \lesssim \frac{m^{2}}{n}$ and a threshold $\kappa_{0}=\kappa_{0}(n, m, r)$, such that if the amplitude of the alignment $\kappa \geqslant \kappa_{0}$, then there is exponential convergence towards flocking,

$$
\left|\mathbf{u}(t, \cdot)-\overline{\mathbf{u}}_{0}\right|_{\infty} \leqslant\left|\delta \mathbf{u}_{0}\right|_{\infty} e^{-\kappa m t}+\frac{2}{m}\left|\delta \mathbf{u}_{0}\right|_{2} e^{-\kappa \eta t} .
$$

Remark 1.2 (On the size of the threshold $\kappa_{0}$ ). Noting that $n m \lesssim \rho\left(\mathcal{S}_{0}\right)=1$, it follows that the threshold $\kappa_{0}$ specified in (4.11) below, does not exceed

$$
\kappa_{0} \lesssim \max \left\{\left|\delta \mathbf{u}_{0}\right|_{\infty},\left|\delta \mathbf{u}_{0}\right|_{2}\right\} \frac{n}{m^{3} r}=C_{0} \times \frac{1}{m^{4} r}, \quad C_{0}=\max \left\{\left|\delta \mathbf{u}_{0}\right|_{\infty},\left|\delta \mathbf{u}_{0}\right|_{2}\right\} .
$$

In the generic case that the initial support $\mathcal{S}_{0}$ is convex, the length of a connecting chain at scale $r$ does not exceed the ratio $n \lesssim \frac{D_{\mathcal{S}_{0}}}{r}$ and we end up with a slightly improved bound

$$
\kappa_{0} \lesssim C_{0} \times \frac{D_{\mathcal{S}_{0}}}{m^{3} r^{2}}, \quad r \lesssim \frac{1}{6} D_{\phi}
$$

Remark 1.3 (Local vs global communication). We note that if the communication kernel $\phi$ has a finite support yet it maintains global communication at $t=0$, that is, if $D_{\phi}>D_{\mathcal{S}_{0}}$ then this yields conditional flocking provided $\kappa$ is assumed to be large enough; this follows from tracing the decreasing Ha-Liu functional, [11],

$$
|\delta \mathbf{u}(t)|_{\infty}+\kappa \int_{D_{\mathcal{S}_{0}}}^{\operatorname{diam}(\mathcal{S}(t))} \phi(h) \mathrm{d} h \leqslant|\delta \mathbf{u}(0)|_{\infty}
$$

The focus of theorems 1.1 and 1.5, however, is in short-range communication where

$$
D_{\phi}<D_{\mathcal{S}_{0}}
$$

The necessity of large enough $\kappa$ in such local cases is clear: otherwise, it is easy to produce an example - we do it in section 6 below, where agents can leave their sensitivity regions in a finite time and then move independently with constant velocities. Observe that a straightforward application of (1.12) to the re-scaled system $(1.1),(t, \mathbf{u}) \mapsto(\kappa t, \mathbf{u} / \kappa)$, would fall short to imply that $\operatorname{diam}(\mathcal{S}(t))$ remains bounded (and hence flocking) since $\phi(h)$ vanishes for $h>D_{\mathcal{S}_{0}}$ in the case of short-range communication (1.13). 
Remark 1.4 (Beyond almost aligned dynamics). The arguments behind theorem 1.1 do not require any smoothness beyond the existence of a classical flow map, $\left\{\mathbb{X}(t, \mathbf{x}) \mid \frac{\mathrm{d}}{\mathrm{d} t} \mathbb{X}(t, \mathbf{x})=\right.$ $\mathbf{u}(t, \mathbb{X}(t, \mathbf{x}))\}$ and $\rho_{0}$ being a non-negative measure, but otherwise, the resulting flocking estimate (1.10) does not depend on higher $L^{p}$ bound of $\rho_{0}$ nor on the higher regularity of $\mathbf{u}$. Our result, therefore, cannot be derived by rescaling the smoothness arguments developed for the almost aligned regimes in [22] and [6].

1.3. Alignment in discrete dynamics. The measure $\rho_{0}$ is only required to have a positive mass on average (over balls at scale $\geqslant \frac{r}{100}$ - consult (3.3) below) but otherwise vacuum is allowed at smaller scales. Consequently, the arguments of theorem 1.1 apply, mutatis mutandis, to deal with $\rho_{0}$ consisting of finitely many Dirac masses which are pushed forward along particle paths. This brings us to our second main result, which addresses the flocking of the discrete setup (1.1) with short-range communication.

Theorem 1.5 (Flocking of CS model). Let $(\mathbf{x}(t), \mathbf{v}(t)), \mathbf{x}=\left(\mathbf{x}_{1}, \ldots, \mathbf{x}_{N}\right), \mathbf{v}=\left(\mathbf{v}_{1}, \ldots, \mathbf{v}_{N}\right)$, be a classical solution of the CS agent-based system (1.1),(1.8) subject to the initial data $\left(\mathbf{x}_{0}, \mathbf{v}_{0}\right)$. Assume that $\mathbf{x}_{0}$ is chain connected at scale $r, r \lesssim \frac{1}{6} D_{\phi}$. Then, there exists a constant $\kappa_{0}$

$$
\kappa_{0}:=\frac{16 N^{4}}{r}\left|\delta \mathbf{v}_{0}\right|_{2}, \quad r \lesssim \frac{1}{6} D_{\phi},
$$

such that for $\kappa \gtrsim \kappa_{0}$ the velocity fluctuation decays exponentially towards a flock, i.e., we have

$$
\left|\mathbf{v}(t, \cdot)-\overline{\mathbf{v}}_{0}\right|_{\infty} \leqslant\left|\delta \mathbf{v}_{0}\right|_{\infty} e^{-\frac{\kappa}{N} t}+2 N \cdot\left|\delta \mathbf{v}_{0}\right|_{2} e^{-\frac{\kappa}{N^{3}} t} .
$$

Remark 1.6 (Dynamics in discrete clusters). The discrete threshold (1.14) follows from theorem 1.1 with $n=N$ Dirac masses, each scaled with mass $m=1 / N$. But we observe that the mass of a ball with radius $r$ centered at one of the $\mathbf{x}_{j}(0)$ 's may in fact be much larger than $1 / N$, if that ball encloses a crowd of Dirac masses. In this case the threshold bound of order $\kappa_{0} \sim n m^{-3}$ improves with growing $m$. In particular, consider initial configurations where the $N$ agents can be partitioned into clusters, $\left\{\mathcal{N}_{\alpha}\right\}_{\alpha}$ of size $n_{\alpha}$, which partition $\{1,2, \ldots, N\}$, such that the agents in each initial cluster are in direct communication

$$
\mathcal{C}_{\alpha}:=\left\{x_{i}(0) \mid i \in \mathcal{N}_{\alpha}\right\}, \quad n_{\alpha}=\# \mathcal{N}_{\alpha}, \quad \sum_{\alpha} n_{\alpha}=N .
$$

Thus, the crowd in cluster $\mathcal{C}_{\alpha}$ is inside a ball of one of its members, say $\mathcal{C}_{\alpha} \subset B_{r}\left(x_{i_{\alpha}}(0)\right)$ with $r \lesssim \frac{1}{6} D_{\phi}$. The mass inside a fixed fraction of each such ball is of order $m \gtrsim \frac{n_{\alpha}}{N}$, and in particular,

$$
m \gtrsim \frac{n_{-}}{N}, \quad n_{-}:=\min _{\alpha} n_{\alpha} .
$$

The number of such clusters does not exceed $n \leqslant N / n_{-}$and we end up with a threshold of order $\kappa_{0} \gtrsim\left(N / n_{-}\right)^{4}$. We conclude the following.

Corollary 1.7 (Finitely many clusters). Let $(\mathbf{x}(t), \mathbf{v}(t))$, be a classical solution of the CS agent-based system (1.1),(1.8) subject to the initial data $\left(\mathbf{x}_{0}, \mathbf{v}_{0}\right)$ which can be partitioned into clusters $\mathcal{C}_{\alpha}$ of size $n_{\alpha}$ with diameter $\lesssim 2 D_{\phi}$,

$$
\mathbf{x}(0) \subset \bigcup_{\alpha} \mathcal{C}_{\alpha}, \quad \operatorname{diam}\left(\mathcal{C}_{\alpha}\right) \lesssim 2 D_{\phi}, \quad n_{\alpha}:=\#\left\{j \mid \mathbf{x}_{j}(0) \in \mathcal{C}_{\alpha}\right\}
$$


Assume these clusters $\left\{\mathcal{C}_{\alpha}\right\}$ are bounded away from vacuum in the sense that $n_{\alpha} / N \geqslant \theta>0$ (and thus, in particular, there are at most $1 / \theta$ of them). Then the flocking threshold depends solely on the finite number of clusters, $\kappa_{0} \lesssim \theta^{-4}$, but otherwise it does not grow with $N$.

The proof of theorems 1.1 (and likewise 1.5) is argued as follows. Let $u_{+}(t)$ and $u_{-}(t)$ denote (scalar) components of the velocity field $\mathbf{u}(t)$ which achieve maximal, and respectively, minimal values at time $t$. Then $u_{ \pm}(t)$ will be attracted to align with their respective local averages, say $\bar{u}_{ \pm}(t)$, localized to the corresponding $D_{\phi}$-neighborhoods of $u_{ \pm}(t)$. Thus, the diameter $D_{u(t)}:=\left|u_{+}(t)-u_{-}(t)\right|$ is expected to decay. However, the decay rate of $D_{u(t)}$ need not be strictly negative: as noted in [26, theorem 3.2], there is a possibility of near constancy (flattening) of $u$ in the neighborhoods of the extrema $u_{ \pm}(t)$. In this case, one needs to extend the first two neighborhoods into a second set of neighborhoods of averages and so, thus creating a global chain of balls of local neighborhoods, connecting $\bar{u}_{+}(t)$ with $\bar{u}_{-}(t)$. This is outlined in section 3. In proposition 4.1 we show that the global variation over such chains is controlled by exponential decay of the fluctuations of the velocity $|\delta \mathbf{u}(t)|_{2}$, which in turn implies, proposition 4.2, exponential decay of the uniform fluctuations $|\delta \mathbf{u}(t)|_{\infty}$. The key question is whether chain connectivity at the local communication scale $D_{\phi}$ persists over time, to facilitate the above argument. To this end we show, in proposition 4.4, that large enough $\kappa$ guarantees that the chain property at scale $\sim D_{\phi}$ persists in time, and hence shorttime process can be prolonged indefinitely thanks to the previously obtained local decay of $|\delta \mathbf{u}(t)|_{\infty}$.

\section{Staying UNIFORMLY AWAY FROM VACUUM}

As noted above, theorem 1.1 does not rule out vacuum in small balls (with scale smaller than $r / 100$ ); in fact, all that we evolve are averaged masses. It is known, however, that such uniform bound is intimatley related to the existence of strong (smooth) solution; see e.g., [28, 24], and earlier results for non-uniform bounds in [7, 23]. Here we show that our arguments can be adapted to obtain such a uniform bound of the one-dimensional density $\rho$ away from 0 in $\Omega=\mathbb{T}$. We extend the techniques used in [24] which cannot be directly used with short range interaction kernels, proving in section 5 the following.

Corollary 2.1. Let $(\rho, u)$ be a strong solution of the $1 D$ system (1.2) on the torus $\mathbb{T}$ subject to non-vacuous data $\left(\rho_{0} \in L^{1}(\mathbb{T}), u_{0} \in C^{1}(\mathbb{T})\right), \rho_{0}>0$, and satisfying the assumption 3.2. Then, there exists a positive constant $C_{0}$ such that

$$
\rho(t, \mathbf{x}) \geqslant C_{0}>0 \quad \text { for all } x \in \mathbb{T}, t \geqslant 0 .
$$

The emergence of flocking for short-range interactions was treated in a few previous works. The first, Shvydkoy-Tadmor [26], in which they consider a short-range topological interactions. In [10], S.-Y. Ha et. al. study the flocking of the one-dimensional CS particle system 1.1 providing explicit critical coupling strength required for the emergence of flocking. C. Jin in [15] treated the Mostch-Tadmor agent-based system [16] with short-range communication, proving flocking under small perturbation of regular-stochastic matrices.

\section{ChAin CONNECTIVITY}

The global flocking analysis of system (1.2) and its underlying discrete version (1.1) driven by compactly supported communication kernel $\phi$ requires a localization procedure. To this end we note that the key local scale in the dynamics of these systems is the communication 
range $1 / 2 D_{\phi}$, where we shall often utilize the following basic observation, namely - if two sets $A, B \subset \Omega$ overlap a non-empty intersection, each with diameter $\operatorname{diam}(A), \operatorname{diam}(B) \leqslant 1 / 2 D_{\phi}$, then their entries are in direct communication so that $\inf _{\mathbf{x} \in A, \mathbf{y} \in B} \phi(|\mathbf{x}-\mathbf{y}|) \geqslant 1$. We then perform a localization procedure based in chain connectivity of the initial support, $\mathcal{S}_{0}$.

Definition 3.1 (Chain connectivity). Fix $r>0$. A bounded domain $\mathcal{D}$ is said to be chain connected at scale $r$ if there exists a finite $n$ (possibly dependent on $r$ ) such that every pair $\mathbf{x}, \mathbf{y} \in \overline{\mathcal{D}}$ is connected through a chain $\mathcal{B}_{\mathbf{x}, \mathbf{y}}$, of at most $n$ balls of radii $r$ with non-empty overlap:

$$
\mathcal{B}_{\mathbf{x}, \mathbf{y}}:=\left\{B_{r}\left(\mathbf{c}_{\alpha}\right)\right\}_{\alpha=1}^{n} \text { such that }\left\{\begin{array}{l}
B_{r}\left(\mathbf{c}_{\alpha}\right) \cap B_{r}\left(\mathbf{c}_{\alpha+1}\right) \neq \emptyset, \quad \mathbf{c}_{\alpha} \in \mathcal{D} \\
\text { connecting } \mathbf{x} \in \bar{B}_{r}\left(\mathbf{c}_{1}\right) \text { and } \mathbf{y} \in \bar{B}_{r}\left(\mathbf{c}_{n}\right) .
\end{array}\right.
$$

Note that the balls $B_{r}\left(\mathbf{c}_{\alpha}\right)$ are not requited to be inside $\mathcal{D}$ - only their centers do, so in the sequel we can also treat discrete domains. A regular, simply-connected domain with a smooth boundary is chain connected. For example, if the boundary of $\mathcal{D}$ satisfies the interior ball condition with scale $r$ (so that each $\mathbf{x} \in \partial \mathcal{D}$ admits a ball $B=B_{r}\left(\mathbf{y}_{\mathbf{x}}\right) \subset \mathcal{D}$ such that $\mathbf{x} \in \partial B$ ) and the interior of $\mathcal{D}$ is arc-connected, then it is chain connected with scale $r$. We shall not dwell on precise characterization of chain-connected domains and simply make the following.

Assumption 3.2 ( $\mathcal{S}_{0}$ is chain connected and non-vacuous). There exists $r>0$ such that the initial support $\mathcal{S}_{0}=\operatorname{supp}\left(\rho_{0}\right)$ satisfies the following.

(i) It is chain connected at scale $r$, namely,

$$
\text { there exist } n \text { balls of diameter } r \text { with } r \leqslant \frac{1}{6} D_{\phi} \text { such that (3.1) holds; }
$$

(ii) All balls with center in $\mathcal{S}_{0}$ with diameter $\frac{r}{100}$ have a positive mass

$$
m=\inf _{B \in \mathcal{B}_{100}} \rho(B)>0, \quad \mathcal{B}_{100}:=\left\{B_{\frac{r}{100}}(\mathbf{c}) \mid \mathbf{c} \in \mathcal{S}_{0}\right\} .
$$

Remark 3.3 (On the length of the chain). Since we did not insist on a minimal chain, its length $n$ (counting the number of balls) is allowed to be large. If we restrict attention to minimal chain, however, then we may assume that each new step of the chain contains a non-negligible increment, say a ball of radius $\frac{r}{100}$

$$
B_{r}\left(\mathbf{c}_{\alpha}\right) \backslash \bigcup_{\beta<\alpha} B_{r}\left(\mathbf{c}_{\beta}\right) \supset B_{\frac{r}{100}}\left(\mathbf{b}_{\alpha}\right) \text { centered around some } \mathbf{b}_{\alpha} \in B_{r}\left(c_{\alpha}\right) .
$$

Then since $\left\{B_{r}\left(\mathbf{b}_{\alpha}\right)\right\}_{\alpha}$ are disjoint and recalling $\rho\left(\mathcal{S}_{0}\right)=1$, we conclude, in view of (3.3), that $n(r) \lesssim \frac{1}{m}$. Alternatively, the cardinality of (minimal) chains depends on the geometry of $\mathcal{S}_{0}$ : for example, if $\mathcal{S}_{0}$ is chord-connected then for 'small' balls, $n(r) \lesssim \frac{D_{\mathcal{S}_{0}}}{r}$. In particular, it follows that each 'small' ball, say, $B_{\frac{r}{100}}(\cdot)$, is covered by at most a fixed ( $r$-independent) number of balls from any chain $\mathcal{B}_{\mathbf{x}, \mathbf{y}}$.

Observe that assumption 3.2 does not require $\rho_{0}$ to be uniformly non-vacuous - only its average over balls above scale $\frac{r}{100}$ need be bounded away from zero (of course, one can replace 100 by another fixed scale $>2$ ). Consequently, the chain connectivity also applies 
in the drastically different scenario of $\rho_{0}$ being a sum of Dirac's masses, which enables us to treat the flocking behavior of both - the discrete agent-based dynamics (1.1) and the continuum hydrodynamics, treated in theorems 1.1 and respectively 1.5 above.

Propagation of connectivity. Let us introduce the key component of our local-to-global approach, in which we propagate the chain connectivity of $\mathcal{S}_{0}$ for the chain connectivity of $\mathcal{S}(t)$. Let $\mathbb{X}^{t}(\mathbf{x})=\mathbb{X}(t, \mathbf{x})$ denote the (forward) flow map,

$$
\frac{\mathrm{d}}{\mathrm{d} t} \mathbb{X}(t, \mathbf{x})=\mathbf{u}(t, \mathbb{X}(t, \mathbf{x})), \quad \text { and } \quad \mathbb{X}(0, \mathbf{x})=\mathbf{x}
$$

It is well known that any smooth solution of the continuity equation preserves the mass of sets along the characteristic flow. That is $\rho\left(t, \mathbb{X}^{t}(A)\right)=\rho_{0}(A)$ for any set $A \subset \mathcal{S}_{0}$ with image $\mathbb{X}^{t}(A)$ along the flow at time $t$. We claim that local propagation of mass ensures that the initial connectivity of $\mathcal{S}_{0}$ is preserved in time. To this end, for any $\mathbf{x}, \mathbf{y} \in \overline{\mathcal{S}}(t)$, we let $\mathbf{x}_{0}={ }^{t} \mathbb{X}(\mathbf{x}) \in \mathcal{S}_{0}$ and $\mathbf{y}_{0}={ }^{t} \mathbb{X}(\mathbf{y}) \in \mathcal{S}_{0}$ denote the pre-images of $\mathbf{x}$ and $\mathbf{y}$, determined by the (backward) flow, abbreviated here as ${ }^{t} \mathbb{X}(\cdot)$. Then by assumption, $\mathbf{x}_{0}$ and $\mathbf{y}_{0}$ are connected through a chain, $\mathcal{B}_{\mathbf{x}_{0}, \mathbf{y}_{0}}=\left\{B_{r}\left(\mathbf{c}_{\alpha}\right)\right\}_{\alpha}$ of scale $r$. Let $\mathcal{F}_{\mathbf{x}, \mathbf{y}}^{t}$ be the image chain by the forward flow

$$
\mathcal{F}_{\mathbf{x}, \mathbf{y}}^{t}=\left\{F_{\alpha}^{t}\right\}_{\alpha=1}^{n}, \quad F_{\alpha}^{t}:=\mathbb{X}^{t}\left(B_{r}\left(\mathbf{c}_{\alpha}\right)\right), \quad \alpha=1,2, \ldots, n .
$$

In this manner we obtain a finite chain $\mathcal{F}_{\mathbf{x}, \mathbf{y}}^{t}$ connecting $\mathbf{x} \in F_{1}^{t}$ to $\mathbf{y} \in F_{n}^{t}$ with intersecting consecutive pairs, $F_{\alpha}^{t} \cap F_{\alpha+1}^{t} \neq \emptyset$.

Thus, the chains $\mathcal{F}_{\mathbf{x}, \mathbf{y}}^{t}$ in (3.5) induce the chain connectivity of $\mathcal{S}(t)$, in analogy to the connectivity of $\mathcal{S}_{0}$ by the chains of balls $\mathcal{B}_{\mathbf{x}_{0}, \mathbf{y}_{0}}$ in (3.1). The essential question is whether the scale of the chain $\mathcal{F}_{\mathbf{x}, \mathbf{y}}^{t}$, measured by $\max \operatorname{diam}\left(F_{\alpha}^{t}\right)$, retains the order of the original scale $r$ of $\mathcal{B}_{\mathbf{x}_{0}, \mathbf{y}_{0}}$. It is here that propagation of alignment properties of the flow play a central role.

\section{Proof of the MAin Results}

The proof of theorems 1.1 and 1.5 relies on three propositions. In the first, proposition 4.1 , we estimate a localized version of velocity fluctuations $|\delta \mathbf{u}|_{2}$ and use the chain connectivity to piece together the local estimates and obtain local-in-time exponential decay of whole $|\delta \mathbf{u}|_{2}$. Then, in Proposition 4.2, we use this decay to prove local-in-time exponential decay of $|\delta \mathbf{u}|_{\infty}$. We need an $L^{\infty}$ decay because, subsequently in Proposition 4.4, we bound the total distance traveled and show that with $\kappa$ large enough, the particles do not escape the range dictated by the essential support of $\phi$. This enables us to prolong the local-in-time estimates and prove the main results.

Exponential energy decay. To prove the exponential decay of the kinetic energy, we introduce the maximal time, $\mathcal{T}=\sup \tau(\kappa)$, during which the flow map does not expand more than $3 r$,

$$
\mathcal{T}:=\sup \left\{\tau>0: \sup _{\substack{\mathbf{x}_{0}, \mathbf{y}_{0} \in \mathcal{S}_{0} \\\left|\mathbf{x}_{0}-\mathbf{y}_{0}\right| \leqslant r}}\left|\mathbb{X}^{\tau}\left(\mathbf{x}_{0}\right)-\mathbb{X}^{\tau}\left(\mathbf{y}_{0}\right)\right| \leqslant 3 r\right\} .
$$

Since by assumption, (1.2) admits a smooth solution, there exists a small enough $\mathcal{T}>0$ such that (4.1) holds. We will show in Proposition 4.4 below, that during that time interval $[0, \mathcal{T}]$, the fluctuations $|\delta \mathbf{u}(t)|_{2}$ decay exponentially. Our objective is then to show that $\mathcal{T}=\infty$ when $\kappa$ is large enough. We achieve this in proposition 4.4 . 
Proposition 4.1. Let $(\rho, \mathbf{u})$ be a strong solution to $(1.2)$ in $[0, \mathcal{T}]$ subject to initial data $\left(\rho_{0}, \mathbf{u}_{0}\right) \in\left(L^{1}\left(\mathcal{S}_{0}\right), C^{1}\left(\mathcal{S}_{0}\right)\right)$ satisfying assumption 3.2. In particular, supp $\left(\rho_{0}\right)$ is chain connected at scale $r \leqslant \frac{1}{6} D_{\phi}$. Then, there exits a constant $\eta=\eta(r, m)>0$, such that

$$
|\delta \mathbf{u}(t)|_{2} \leqslant e^{-\kappa \eta t}\left|\delta \mathbf{u}_{0}\right| \quad \text { for all } t \leqslant \mathcal{T}, \quad \eta \lesssim \frac{m^{2}}{n(r)} .
$$

Remark that in proposition 4.4 below we derive a minimal value of the threshold $\kappa$ which is inversely proportional to $\eta$, hence we will pay attention to tracing upper-bound of the latter in terms of $m$ and $n(r)$.

Proof. We begin by noting that during the time interval $[0, \mathcal{T}]$, the maximal diameter of each element in the connecting chain $F_{\alpha}^{t} \in \mathcal{F}_{\mathbf{x}, \mathbf{y}}^{t}$ cannot expand more than $r$ units beyond the diameter of its pre-image ball $B_{r}\left(\mathbf{c}_{\alpha}\right)$,

$$
\operatorname{diam}\left(F_{\alpha}^{t}\right) \leqslant 3 r, \quad t \leqslant \mathcal{T} .
$$

Moreover, $\rho\left(F_{\alpha}^{t}\right)=\rho\left(B_{r}\left(\mathbf{c}_{\alpha}\right)\right) \geqslant m$. The proof proceeds in three steps.

Step 1 (Restriction). We begin by making a general observation that allows us to restrict attention to fluctuations over sets with diameter width $6 r \leqslant D_{\phi}$. Specifically, we claim that for (4.2) to hold, it suffices to bound energy fluctuations in terms of the restricted enstrophy on stripes of width $\leqslant 6 r$

$$
|\delta \mathbf{u}(t)|_{2}^{2} \leqslant \frac{1}{\eta}|\delta \mathbf{u}(t)|_{2, r}^{2}, \quad|\delta \mathbf{u}(t)|_{2, r}^{2}:=\int_{\mathbf{x} \in \mathcal{S}} \int_{\mathbf{y} \in \mathcal{S} \cap B_{6 r}(\mathbf{x})}|\mathbf{u}(t, \mathbf{x})-\mathbf{u}(t, \mathbf{y})|^{2} \mathrm{~d} \rho(\mathbf{x}) \mathrm{d} \rho(\mathbf{y}) .
$$

Indeed, since $6 r$ does not exceed the communication scale $D_{\phi}$ so that $\phi(|\mathbf{x}-\mathbf{y}|) \geqslant 1$ wherever $\mathbf{y} \in \mathcal{S}(t) \cap B_{6 r}(\mathbf{x})$, the enstrophy bound (1.4) yields

$$
\begin{aligned}
\frac{1}{2} \frac{\mathrm{d}}{\mathrm{d} t}|\delta \mathbf{u}(t)|_{2}^{2} & =-\kappa \int_{\mathcal{S}(t) \times \mathcal{S}(t)} \phi(|\mathbf{x}-\mathbf{y}|)|\mathbf{u}(t, \mathbf{x})-\mathbf{u}(t, \mathbf{y})|^{2} \mathrm{~d} \rho(\mathbf{x}) \mathrm{d} \rho(\mathbf{y}) \\
& \leqslant-\kappa \int_{\mathbf{x} \in \mathcal{S}} \int_{\mathbf{y} \in \mathcal{S}(t) \cap B_{6 r}(\mathbf{x})} \phi(|\mathbf{y}|)|\mathbf{u}(t, \mathbf{x})-\mathbf{u}(t, \mathbf{y})|^{2} \mathrm{~d} \rho(\mathbf{x}) \mathrm{d} \rho(\mathbf{y}) \leqslant-\kappa|\delta \mathbf{u}(t)|_{2, r}^{2},
\end{aligned}
$$

and together with the assumed bound (4.4), we end up with exponential decay rate corresponding to $(4.2)$

$$
\frac{\mathrm{d}}{\mathrm{d} t}|\delta \mathbf{u}(t)|_{2}^{2} \leqslant-2 \kappa|\delta \mathbf{u}(t)|_{2, r}^{2} \leqslant-2 \kappa \eta|\delta \mathbf{u}(t)|_{2}^{2}
$$

Step 2 (Localization). To simplify notation we often suppress the time dependence of quantities evaluated at time $t$, abbreviating $\mathcal{S}=\mathcal{S}(t), \mathbf{u}(\mathbf{x})=\mathbf{u}(t, \mathbf{x}), F_{\alpha}=F_{\alpha}^{t}$ etc. In the sequel we shall work with averages rather than point values: we use $\overline{\mathbf{u}}_{A}$ to denote the average $\overline{\mathbf{u}}_{A}:=\frac{1}{\rho(A)} \int_{A} \mathbf{u}(\mathbf{x}) \mathrm{d} \rho(\mathbf{x})$.

For an arbitrary pair $\mathbf{x}, \mathbf{y} \in \overline{\mathcal{S}}$, we consider their connecting chain $\mathcal{F}_{\mathbf{x}, \mathbf{y}}^{t}=\left\{F_{\alpha}\right\}_{\alpha=1}^{n}$, and express the difference $\mathbf{u}(\mathbf{x})-\mathbf{u}(\mathbf{y})$ in terms of the intermediate averages along that chain,

$$
\mathbf{u}(\mathbf{x})-\mathbf{u}(\mathbf{y}) \equiv\left(\mathbf{u}(\mathbf{x})-\overline{\mathbf{u}}_{1}\right)+\sum_{\alpha=1}^{n-1}\left(\overline{\mathbf{u}}_{\alpha}-\overline{\mathbf{u}}_{\alpha+1}\right)+\left(\overline{\mathbf{u}}_{n}-\mathbf{u}(\mathbf{y})\right), \quad \overline{\mathbf{u}}_{\alpha}:=\overline{\mathbf{u}}_{F_{\alpha}}
$$


This enables us to decompose a global energy fluctuations bound into

$$
\begin{aligned}
& \int_{\mathcal{S} \times \mathcal{S}}|\mathbf{u}(\mathbf{x})-\mathbf{u}(\mathbf{y})|^{2} \mathrm{~d} \rho(\mathbf{x}) \mathrm{d} \rho(\mathbf{y}) \\
& \leqslant(n+1)\left(\int_{\mathbf{y} \in \mathcal{S}} \int_{\mathbf{x} \in \mathcal{S}}\left|\mathbf{u}(\mathbf{x})-\overline{\mathbf{u}}_{1}\right|^{2} \mathrm{~d} \rho(\mathbf{x}) \mathrm{d} \rho(\mathbf{y})\right. \\
& \left.\quad+\int_{\mathcal{S} \times \mathcal{S}} \sum_{\alpha=1}^{n-1}\left|\overline{\mathbf{u}}_{\alpha}-\overline{\mathbf{u}}_{\alpha+1}\right|^{2} \mathrm{~d} \rho(\mathbf{x}) \mathrm{d} \rho(\mathbf{y})+\int_{\mathbf{x} \in \mathcal{S}} \int_{\mathbf{y} \in \mathcal{S}}\left|\mathbf{u}(\mathbf{y})-\overline{\mathbf{u}}_{n}\right|^{2} \mathrm{~d} \rho(\mathbf{y}) \mathrm{d} \rho(\mathbf{x})\right) \\
& =:(n+1)(I+I I+I I I) .
\end{aligned}
$$

Note that the averages $\left\{\overline{\mathbf{u}}_{\alpha}\right\}_{\alpha}$ are dependent on $(\mathbf{x}, \mathbf{y})$, and in particular therefore, the first and third integrands on the right depend on $(\mathbf{x}, \mathbf{y})$ because $\overline{\mathbf{u}}_{1}$ and $\overline{\mathbf{u}}_{n}$ do. It remains to bound the local fluctuations on the right.

Step 3 (Local estimates). We bound the first term first. Since $F_{1} \ni \mathbf{x}$ and since by (4.3) its diameter, at least for $t \leqslant \mathcal{T}$, does not exceed $3 r$, which is less than the communication scale $1 / 2 D_{\phi}$, we have

$$
\begin{aligned}
& \int_{\mathbf{x} \in \mathcal{S}}\left|\mathbf{u}(\mathbf{x})-\overline{\mathbf{u}}_{1}\right|^{2} \mathrm{~d} \rho(\mathbf{x})=\int_{\mathbf{x} \in \mathcal{S}} \frac{1}{\rho^{2}\left(F_{1}\right)}\left|\int_{\mathbf{y}^{\prime} \in F_{1}}\left(\mathbf{u}(\mathbf{x})-\mathbf{u}\left(\mathbf{y}^{\prime}\right)\right) \mathrm{d} \rho\left(\mathbf{y}^{\prime}\right)\right|^{2} \mathrm{~d} \rho(\mathbf{x}) \\
& \leqslant \int_{\mathbf{x} \in \mathcal{S}} \frac{1}{\rho\left(F_{1}\right)} \int_{\mathbf{y}^{\prime} \in \mathcal{S} \cap B_{3 r}(\mathbf{x})}\left|\mathbf{u}(\mathbf{x})-\mathbf{u}\left(\mathbf{y}^{\prime}\right)\right|^{2} \mathrm{~d} \rho\left(\mathbf{y}^{\prime}\right) \mathrm{d} \rho(\mathbf{x}) \leqslant \frac{1}{m}|\delta \mathbf{u}(t)|_{2, r}^{2} .
\end{aligned}
$$

The last bound is uniform with respect to the dependence of $\overline{\mathbf{u}}_{1}$ on $\mathbf{y}$, and since $\mathcal{S}$ has unit mass,

$$
I=\int_{\mathbf{y} \in \mathcal{S}} \int_{\mathbf{x} \in \mathcal{S}}\left|\mathbf{u}(\mathbf{x})-\overline{\mathbf{u}}_{1}\right|^{2} \mathrm{~d} \rho(\mathbf{x}) \mathrm{d} \rho(\mathbf{y}) \leqslant \frac{1}{m}|\delta \mathbf{u}(t)|_{2, r}^{2} .
$$

Similarly, for the third term, $I I I=\int_{\mathbf{x} \in \mathcal{S}} \int_{\mathbf{y} \in \mathcal{S}}\left|\mathbf{u}(\mathbf{y})-\overline{\mathbf{u}}_{n}\right|^{2} \mathrm{~d} \rho(\mathbf{y}) \leqslant \frac{1}{m}|\delta \mathbf{u}(t)|_{2, r}^{2}$.

To estimate the difference of consecutive averages in $I I$, we begin by noting that

$$
\rho(A) \rho(B)\left|\overline{\mathbf{u}}_{A}-\overline{\mathbf{u}}_{B}\right|^{2} \leqslant \int_{\left(\mathbf{x}^{\prime}, \mathbf{y}^{\prime}\right) \in(A \times B)}\left|\mathbf{u}\left(\mathbf{x}^{\prime}\right)-\mathbf{u}\left(\mathbf{y}^{\prime}\right)\right|^{2} \mathrm{~d} \rho\left(\mathbf{x}^{\prime}\right) \mathrm{d} \rho\left(\mathbf{y}^{\prime}\right),
$$

which is nothing but the Cauchy-Schwartz bound for the identity

$$
\overline{\mathbf{u}}_{A}-\overline{\mathbf{u}}_{B}=\int_{\left(\mathbf{x}^{\prime}, \mathbf{y}^{\prime}\right) \in(A \times B)}\left(\mathbf{u}\left(\mathbf{x}^{\prime}\right)-\mathbf{u}\left(\mathbf{y}^{\prime}\right)\right) \frac{\mathrm{d} \rho\left(\mathbf{x}^{\prime}\right) \mathrm{d} \rho\left(\mathbf{y}^{\prime}\right)}{\rho(A) \rho(B)}
$$

Set $A=F_{\alpha}^{t}$ and $B=F_{\alpha+1}^{t}$, and recall that the mass of each is at least $m$; hence

$$
\left|\overline{\mathbf{u}}_{\alpha}-\overline{\mathbf{u}}_{\alpha+1}\right|^{2} \leqslant \frac{1}{m^{2}} \int_{\mathbf{x}^{\prime} \in F_{\alpha+1}} \int_{\mathbf{y}^{\prime} \in F_{\alpha}}\left|\mathbf{u}\left(\mathbf{x}^{\prime}\right)-\mathbf{u}\left(\mathbf{y}^{\prime}\right)\right|^{2} \mathrm{~d} \rho(\mathbf{y}) \mathrm{d} \rho(\mathbf{x}) .
$$

By assumption, $F_{\alpha}$ and $F_{\alpha+1}$ (which still depend on $(\mathbf{x}, \mathbf{y})$ ) have a non-empty intersection hence (4.3) implies for $\left(\mathbf{x}^{\prime}, \mathbf{y}^{\prime}\right) \in\left(F_{\alpha+1}^{t} \times F_{\alpha}^{t}\right) \leadsto\left|\mathbf{y}^{\prime}-\mathbf{x}^{\prime}\right| \leqslant \operatorname{diam}\left(F_{\alpha+1}^{t}\right)+\operatorname{diam}\left(F_{\alpha}^{t}\right) \leqslant 6 r$,

$$
\left|\overline{\mathbf{u}}_{\alpha}-\overline{\mathbf{u}}_{\alpha+1}\right|^{2} \leqslant \frac{1}{m^{2}} \int_{\mathbf{x}^{\prime} \in F_{\alpha+1}} \int_{\mathbf{y}^{\prime} \in \mathcal{S} \cap B_{6 r}\left(\mathbf{x}^{\prime}\right)}\left|\mathbf{u}\left(\mathbf{x}^{\prime}\right)-\mathbf{u}\left(\mathbf{y}^{\prime}\right)\right|^{2} \mathrm{~d} \rho\left(\mathbf{y}^{\prime}\right) \mathrm{d} \rho\left(\mathbf{x}^{\prime}\right) .
$$


We note that although the elements in the chain $\mathcal{F}_{\mathbf{x}, \mathbf{y}}^{t}$ have non-empty overlaps, the assumed minimality of the chain implies that the union $\cup_{\alpha} F_{\alpha}^{t}$ can cover $\mathcal{S}(t)$ only finitely many times (see remark 3.3 above). Consequently, the second term does not exceed (recall $\rho(\mathcal{S}(t)) \equiv 1$ )

$$
\begin{aligned}
I I & =\int_{\mathcal{S} \times \mathcal{S}} \sum_{\alpha=1}^{n-1}\left|\overline{\mathbf{u}}_{\alpha}-\overline{\mathbf{u}}_{\alpha+1}\right|^{2} \mathrm{~d} \rho(\mathbf{x}) \mathrm{d} \rho(\mathbf{y}) \\
& \lesssim \frac{1}{m^{2}} \int_{\mathbf{x}^{\prime} \in \mathcal{S}} \int_{\mathbf{y}^{\prime} \in \mathcal{S} \cap B_{6 r}\left(\mathbf{x}^{\prime}\right)}\left|\mathbf{u}\left(\mathbf{x}^{\prime}\right)-\mathbf{u}\left(\mathbf{y}^{\prime}\right)\right|^{2} \mathrm{~d} \rho\left(\mathbf{y}^{\prime}\right) \mathrm{d} \rho\left(\mathbf{x}^{\prime}\right) \leqslant \frac{1}{m^{2}}|\delta \mathbf{u}(t)|_{2, r}^{2} .
\end{aligned}
$$

We conclude that (4.4) holds with $\eta \lesssim \frac{m^{2}}{n(r)}$ :

$$
|\delta \mathbf{u}(t)|_{2}^{2} \lesssim(n+1)\left(\frac{2}{m}+\frac{1}{m^{2}}\right)|\delta \mathbf{u}(t)|_{2, r}^{2}
$$

and (4.2) follows.

Bounding the diameter of velocity field. The next proposition provides an estimate of the maximal velocity fluctuations, $|\delta \mathbf{u}|_{\infty}$, in terms of the kinetic energy and coefficients that decay exponentially.

Proposition 4.2. Let $(\rho, \mathbf{u})$ be a strong solution to (1.2) in $[0, \mathcal{T}]$ subject to initial data $\left(\rho_{0}, \mathbf{u}_{0}\right) \in\left(L^{1}\left(\mathcal{S}_{0}\right), C^{1}\left(\mathcal{S}_{0}\right)\right)$ with a chain connected support supp $\left(\rho_{0}\right)$ of scale $r \leqslant \frac{1}{6} D_{\phi}$. Then we have

$$
|\delta \mathbf{u}(t)|_{\infty} \leqslant\left|\delta \mathbf{u}_{0}\right|_{\infty} e^{-\kappa m t}+\kappa \int_{0}^{t} e^{-\kappa m(t-\sigma)}|\delta \mathbf{u}(\sigma)|_{2} d \sigma, \quad t \leqslant T .
$$

Proof. We first employ the argument of [17, theorem 2.3], [14, Sec. 1] in order to restrict attention to a scalar projection of $\mathbf{u}$ : fix an arbitrary unit vector $\mathbf{w}$, then a projection of (1.2) on w yields

$$
\partial_{t}\langle\mathbf{u}, \mathbf{w}\rangle+\mathbf{u} \cdot \nabla_{\mathbf{x}}\langle\mathbf{u}, \mathbf{w}\rangle=\kappa \int_{\mathcal{S}(t)} \phi(|\mathbf{x}-\mathbf{y}|)(\langle\mathbf{u}(\mathbf{y}), \mathbf{w}\rangle-\langle\mathbf{u}(\mathbf{x}), \mathbf{w}\rangle) \mathrm{d} \rho(\mathbf{y}) .
$$

Let $\mathbf{u}_{ \pm}(t)$ be the extremal projected values, $\left\langle\mathbf{u}_{ \pm}, \mathbf{w}\right\rangle=\left\{\begin{array}{c}\max _{\mathcal{S}(t)} \\ \min _{\mathcal{S}(t)}\end{array}\right\}\langle\mathbf{u}, \mathbf{w}\rangle$ at $\mathbf{x}_{+}=\mathbf{x}_{+}(t)$ and, respectively, at $\mathbf{x}_{-}=\mathbf{x}_{-}(t)^{3}$. Let $F_{+}^{t} \ni \mathbf{x}_{+}$and $F_{-}^{t} \ni \mathbf{x}_{-}$be the two sets from the chain $\mathcal{F}^{t}$ covering $\mathcal{S}(t)$. Since $\operatorname{diam}\left(\mathcal{F}_{ \pm}^{t}\right) \leqslant 3 r \leqslant 1 / 2 D_{\phi}$,

$$
\begin{aligned}
& \frac{\mathrm{d}}{\mathrm{d} t}\left\langle\mathbf{u}_{+}, \mathbf{w}\right\rangle=\kappa \int_{\mathcal{S}(t)} \phi\left(\left|\mathbf{x}_{+}-\mathbf{y}\right|\right)\left(\langle\mathbf{u}(\mathbf{y}), \mathbf{w}\rangle-\left\langle\mathbf{u}_{+}, \mathbf{w}\right\rangle\right) \mathrm{d} \rho(\mathbf{y}) \leqslant \kappa \int_{F_{+}^{t}}\left(\langle\mathbf{u}(\mathbf{y}), \mathbf{w}\rangle-\left\langle\mathbf{u}_{+}, \mathbf{w}\right\rangle\right) \mathrm{d} \rho(\mathbf{y}), \\
& \frac{\mathrm{d}}{\mathrm{d} t}\left\langle\mathbf{u}_{-}, \mathbf{w}\right\rangle=\kappa \int_{\mathcal{S}(t)} \phi\left(\left|\mathbf{x}_{-}-\mathbf{y}\right|\right)\left(\langle\mathbf{u}(\mathbf{y}), \mathbf{w}\rangle-\left\langle\mathbf{u}_{-}, \mathbf{w}\right\rangle\right) \mathrm{d} \rho(\mathbf{y}) \geqslant \kappa \int_{F_{-}^{t}}\left(\langle\mathbf{u}(\mathbf{y}), \mathbf{w}\rangle-\left\langle\mathbf{u}_{-}, \mathbf{w}\right\rangle\right) \mathrm{d} \rho(\mathbf{y}),
\end{aligned}
$$

\footnotetext{
${ }^{3} \mathbf{x}_{ \pm}$need not be unique - any extreme location will suffice.
} 
and since the mass of $F_{ \pm}^{t}$ is at least $m$, we control the velocity diameter $D_{\mathbf{u}(t)}:=\mathbf{u}_{+}(t)-\mathbf{u}_{-}(t)$ projected on $\mathbf{w}$,

$$
\begin{aligned}
\frac{\mathrm{d}}{\mathrm{d} t} & \left\langle D_{\mathbf{u}(t)}, \mathbf{w}\right\rangle \\
& \leqslant \kappa \frac{m}{\rho\left(F_{+}^{t}\right)} \int_{F_{+}^{t}}\left(\langle\mathbf{u}(\mathbf{y}), \mathbf{w}\rangle-\left\langle\mathbf{u}_{+}, \mathbf{w}\right\rangle\right) \mathrm{d} \rho(\mathbf{y})-\kappa \frac{m}{\rho\left(F_{-}^{t}\right)} \int_{F_{-}^{t}}\left(\langle\mathbf{u}(\mathbf{y}), \mathbf{w}\rangle-\left\langle\mathbf{u}_{-}, \mathbf{w}\right\rangle\right) \mathrm{d} \rho(\mathbf{y}) \\
& =-\kappa m\left\langle D_{\mathbf{u}(t)}, \mathbf{w}\right\rangle+\kappa m\left\langle\overline{\mathbf{u}}_{F_{+}^{t}}-\overline{\mathbf{u}}_{F_{-}^{t}}, \mathbf{w}\right\rangle, \quad\left\langle D_{\mathbf{u}(t)}, \mathbf{w}\right\rangle=\left\langle\mathbf{u}_{+}(t)-\mathbf{u}_{-}(t), \mathbf{w}\right\rangle .
\end{aligned}
$$

By (4.5), $m\left|\overline{\mathbf{u}}_{F_{+}^{t}}-\overline{\mathbf{u}}_{F_{-}^{t}}\right|$ does not exceed $|\delta \mathbf{u}|_{2}$, hence

$$
\frac{\mathrm{d}}{\mathrm{d} t}\left\langle D_{\mathbf{u}(t)}, \mathbf{w}\right\rangle \leqslant-\kappa m\left\langle D_{\mathbf{u}(t)}, \mathbf{w}\right\rangle+\kappa|\delta \mathbf{u}(t)|_{2} .
$$

Integration yields $\left\langle D_{\mathbf{u}(t)}, \mathbf{w}\right\rangle \leqslant\left\langle D_{\mathbf{u}_{0}}, \mathbf{w}\right\rangle e^{-\kappa m t}+\kappa \int_{0}^{t} e^{-\kappa m(t-\sigma)}|\delta \mathbf{u}(\sigma)|_{2} d \sigma$, and taking the supremum over all unit w's recovers the desired bound (4.8) for $|\delta \mathbf{u}(t)|_{\infty}=\max _{|\mathbf{w}|=1}\left\langle D_{\mathbf{u}(t)}, \mathbf{w}\right\rangle$.

Remark 4.3. Observe that the decay rate for the diameter $\left\langle D_{\mathbf{u}(t)}, \mathbf{w}\right\rangle$ in (4.9) need not be strictly negative: as noted in [26, theorem 3.2], there is a possibility of constancy (flattening) of $\mathbf{u}(t)$ in the $F_{ \pm}^{t}$-neighborhoods of the extrema $\mathbf{u}_{ \pm}(t)$, where the RHS of (4.9) cannot be bounded away from $0-$. Instead, this is circumvented here by plugging the known exponential decay of the energy (4.2) into (4.8) to conclude

$$
\begin{aligned}
|\delta \mathbf{u}(t)|_{\infty} & \leqslant\left|\delta \mathbf{u}_{0}\right|_{\infty} e^{-\kappa m t}+\kappa \cdot\left|\delta \mathbf{u}_{0}\right|_{2} \cdot e^{-\kappa m t} \int_{0}^{t} e^{\kappa(m-\eta) \sigma} d \sigma \\
& \leqslant\left|\delta \mathbf{u}_{0}\right|_{\infty} \cdot e^{-\kappa m t}+K \cdot\left|\delta \mathbf{u}_{0}\right|_{2} \cdot e^{-\kappa \eta t}, \quad K:=\frac{1}{m-\eta} \leqslant \frac{2}{m} .
\end{aligned}
$$

Bound on the total distance traveled. Here we show that with large enough $\kappa$, the time interval $[0, \mathcal{T}]$ used in Propositions 4.1 and 4.2 can be extended indefinitely.

Proposition 4.4 (Proof of theorem 1.1). Let $(\rho, \mathbf{u})$ be a strong solution to $(1.2)$ in $[0, \mathcal{T}]$ subject to initial data $\left(\rho_{0}, \mathbf{u}_{0}\right) \in\left(L^{1}\left(\mathcal{S}_{0}\right), C^{1}\left(\mathcal{S}_{0}\right)\right)$ satisfying assumption 3.2. In particular, the supp $\left(\rho_{0}\right)$ is assumed to be chain connected at scale $r \leqslant \frac{1}{6} D_{\phi}$. Further, assume that the amplitude of alignment is large enough

$$
\kappa \geqslant \kappa_{0}:=\frac{1}{r m}\left(\frac{1}{2}\left|\delta \mathbf{u}_{0}\right|_{\infty}+\frac{1}{\eta}\left|\delta \mathbf{u}_{0}\right|_{2}\right), \quad \eta \lesssim \frac{m^{2}}{n(r)} .
$$

Then the chain connectivity of the associated flow map in (4.1) persists for all time, $\mathcal{T}=$ $[0, \infty)$ and theorem 1.1 with (1.11) follows.

Indeed, integrating the fluctuations of $(3.4), \frac{\mathrm{d}}{\mathrm{d} t} \delta \mathbb{X}^{t}(\cdot)=\delta \mathbf{u}(t, \cdot)$, and utilizing (4.10) we find the uniform-in- $\tau$ bound

$$
\left|\mathbb{X}^{\tau}\left(\mathbf{x}_{0}\right)-\mathbb{X}^{\tau}\left(\mathbf{y}_{0}\right)\right| \leqslant\left|\mathbf{x}_{0}-\mathbf{y}_{0}\right|+\int_{0}^{\tau}|\delta \mathbf{u}(t)|_{\infty} \mathrm{d} t<\left|\mathbf{x}_{0}-\mathbf{y}_{0}\right|+\frac{\left|\delta \mathbf{u}_{0}\right|_{\infty}}{\kappa m}+\frac{2}{m} \cdot \frac{\left|\delta \mathbf{u}_{0}\right|_{2}}{\kappa \eta}, \quad \tau<\infty .
$$

Thus, large enough $\kappa,(4.11)$, guarantees that initial fluctuations of size $\left|\mathbf{x}_{0}-\mathbf{y}_{0}\right| \leqslant r$ do not expand more than $\left|\mathbb{X}^{\tau(\kappa)}\left(\mathbf{x}_{0}\right)-\mathbb{X}^{\tau(\kappa)}\left(\mathbf{y}_{0}\right)\right| \leqslant 3 r$ for all $\tau$ 's, and hence the maximal time of connectivity $\mathcal{T}(\kappa):=\sup \tau(\kappa)=\infty$, proving the exponential flocking asserted in theorem 1.1. 
Remark 4.5 (Large communication scale). Observe that if the essential support of $\phi$ is large, $D_{\phi} \gg 1$, then $\kappa_{0}$ with $r \gg 1$ (recall $m \gtrsim 1$ and hence $n(r) \lesssim 1$ ) recovers unconditional flocking provided

$$
\min _{h \leqslant r} \phi(h) r \stackrel{r \rightarrow \infty}{\longrightarrow} \infty,
$$

which is only slightly stronger than the 'fat tail' condition (1.5).

Proof of the discrete case (theorem 1.5). We consider the discrete solution $\rho_{0}=$ $\frac{1}{N} \sum_{j=1}^{N} \delta_{\mathbf{x}_{j}(0)}(\mathbf{x})$, with the discrete support $\mathcal{S}_{0}=\left\{\mathbf{x}_{j}(0)\right\}_{j=1}^{N}$, normalized to have a unit mass so that (1.3) holds. The assumed chain connectivity of the discrete $\mathcal{S}_{0}$ involves no more than $n=N$ balls with diameters $r$, connecting every initial pair $\left(\mathbf{x}_{i}(0), \mathbf{x}_{j}(0)\right)$. The average mass of each $r$-ball centered $\mathcal{S}_{0}$ - that is, at one of the $\mathbf{x}_{j}(0)$ 's has mass $\geqslant m=\frac{1}{N}$ so that we maintain the counting bound $n m \lesssim 1$. With this we recover the bound (4.11) with $\eta \lesssim 1 / N^{3}\left(\right.$ and since $\left.N\left|\delta \mathbf{v}_{0}\right|_{2}>\left|\delta \mathbf{v}_{0}\right|_{\infty}\right)$

$$
\kappa_{0} \lesssim \frac{N^{4}}{r}\left|\delta \mathbf{v}_{0}\right|_{2}
$$

\section{UNIFORMLY BOUNDED DENSITY AWAY FROM VACUUM}

The density in (1.2) plays a central role as the carrier of local averaging, and hence a uniform bound on the density $\rho(t, \cdot)$ away from vacuum is essential for the existence of strong solutions to (1.2) and their asymptotic behavior. This is particularly relevant in the case of singular interaction kernels, [23, 24, 25, 7, 26]. As noted in [26], the lower bound $\rho \gtrsim 1 / \sqrt{1+t}$ will suffice to yield unconditional flocking in the general multiD case. In $[23,7,26]$ it was shown that the $1 \mathrm{D}$ density is controlled from below, $\rho \geqslant 1 /(1+t)$, while in [24] this estimate was improved to a uniform bound $\rho \geqslant c_{0}>0$. In all cases, the bounds depend on the long-range support of $\phi$. Here we treat the case of short-range kernels, proving the uniform bound away from vacuum by adapting our chain connectivity to the proof [24, Lemma 3.1].

Proof of Corollary 2.1. As in [24], we use the quantity $q$

$$
q(t, x):=\frac{1}{\rho(t, y)}\left(u_{x}(t, x)+\kappa \int_{y \in \mathbb{T}} \phi(|x-y|)(\rho(t, y)-\rho(t, x)) d y\right)
$$

to rewrite the mass equation $(1.2)_{1}$ in an equivalent diffusive form (abbreviating $\rho(t, \cdot)=\rho(\cdot)$ )

$$
\rho_{t}(x)+u \rho_{x}(x)=-q \rho^{2}(x)+\kappa \rho(x) \int_{\mathbb{T}} \phi(|x-y|)(\rho(y)-\rho(y)) \mathrm{d} y .
$$

Evaluating the above expression at $\rho_{-}(t)=\min _{x \in \mathbb{T}} \rho(t, x)$ leads to

$$
\frac{\mathrm{d}}{\mathrm{d} t} \rho_{-}=-q \rho_{-}^{2}+\kappa \rho_{-} \int_{\mathbb{T}} \phi\left(\left|x_{-}-y\right|\right)\left(\rho(y)-\rho_{-}\right) \mathrm{d} y \geqslant-q \rho_{-}^{2}+\kappa \underbrace{\kappa \rho_{-} \int_{F_{-}^{t}} \phi\left(\left|x_{-}-y\right|\right)\left(\rho(y)-\rho_{-}\right) \mathrm{d} y}_{\geqslant 0} .
$$

Here $x_{-}=x_{-}(t)$ is any point at which $\rho(t)$ attains its minimum, and $F_{-}^{t} \in \mathcal{F}$ is any local neighborhood surrounding $x_{-} \in F_{-}^{t}$. Recall that according to (4.3), the diameter $\operatorname{diam}\left(F_{-}^{t}\right) \leqslant 3 r<1 / 2 D_{\phi}$ and hence $\phi\left(\left|x_{-}-y\right|\right) \geqslant 1$ for $y \in F_{-}^{t}$. Thus, since $\rho\left(F_{-}^{t}\right) \geqslant m$,

$$
\frac{\mathrm{d}}{\mathrm{d} t} \rho_{-} \geqslant-q \rho_{-}^{2}+\kappa \rho_{-} \cdot \int_{F_{-}^{t}} \phi\left(\left|x_{-}-y\right|\right)\left(\rho(\mathbf{y})-\rho\left(x_{-}\right)\right) \mathrm{d} y \geqslant-q \rho_{-}^{2}+\kappa \rho_{-} \cdot m-\rho_{-}^{2} \kappa\left|F_{-}^{t}\right|,
$$


where the volume of $F_{-}^{t}$ is bounded since its diameter is. We end up with

$$
\frac{\mathrm{d}}{\mathrm{d} t} \rho_{-} \gtrsim-\left(|q|_{\infty}+\kappa\right) \rho_{-}^{2}+\kappa m \cdot \rho_{-} .
$$

What signifies the quantity $q$ is that it is being transported, $q_{t}+u q_{x}=0$, e.g., [23, $\left.\mathbb{S} 2.2\right]$, hence $|q|_{\infty} \leqslant\left|q_{0}\right|_{\infty}$, and we conclude with a uniform lower-bound (2.1)

$$
\min _{x \in \mathbb{T}} \rho(t, x) \gtrsim \min \left\{\rho_{-}(0), \frac{\kappa m}{\left|q_{0}\right|_{\infty}+\kappa}\right\}>0 .
$$

\section{Closing Remarks}

While we do not claim optimality of the threshold $\kappa_{0}$, we observe that the existence of such threshold is required: it is easy to construct a counterexample where the intensity of the interaction $\kappa$ is not sufficient to ensure flocking for given initial condition and $D_{\phi}$. Indeed, let us consider a bounded communication weight satisfying (1.8) with $D_{\phi}=4$ and two particles on a line with $x_{1}(0)=-1, x_{2}(0)=1, v_{1}(0)=-1, v_{2}(0)=1$. Then, putting

$$
x(t)=x_{2}(t)-x_{1}(t)>0, \quad v(t)=v_{2}(t)-v_{1}(t)>0,
$$

we reformulate system (1.1) into

$$
\dot{x}=v, \quad \dot{v}=-2 \kappa v \phi(x)=-2 \kappa \frac{\mathrm{d}}{\mathrm{d} t} \Phi(x), \quad \Phi(r):=\int_{0}^{r} \phi(h) \mathrm{d} h .
$$

Integration of (6.1) leads to

$$
\begin{aligned}
& v(t)=2 \kappa \Phi(x(0))-2 \kappa \Phi(x(t))+v(0) \\
& x(t)=2 \kappa t \Phi(x(0))-2 \kappa \int_{0}^{t} \Phi(x(t)) \mathrm{d} t+t v(0)+x(0) .
\end{aligned}
$$

Since $\phi$ is bounded and 0 outside of, say, $\left[0,2 D_{\phi}\right]$ then $\Phi$ is also bounded. Therefore, as long as $x \leqslant 2 D_{\phi}=8$ we have

$$
x(t) \geqslant-8 \kappa t|\Phi|_{\infty}+t v(0)+x(0) .
$$

Now, if $\kappa \leqslant v(0) / 16|\Phi|_{\infty}$ then $x(t) \geqslant \frac{t}{2} v(0)+x(0)$ and $x$ grows steadily with the rate that can be bounded from below by $t v(0) / 2$ and eventually reaches $2 D_{\phi}=8$. As soon as this happens $\phi(x)=0$, and the particles move away from each other with a constant velocity. A similar example can be produced for singular $\phi$.

\section{REFERENCES}

[1] José A. Carrillo, Young-Pil Choi, Piotr B. Mucha Jan and Peszek, Sharp conditions to avoid collisions in singular Cucker-Smale interactions, Nonlinear Analysis 37: 317-328, 2017.

[2] J. A. Carrillo, M. Fornasier, G. Toscani, and F. Vecil. Particle, kinetic, and hydrodynamic models of swarming, 2010. in Mathematical Modeling of Collective Behavior in Socio-Economic and Life Sciences (G. Naldi L. Pareschi G. Toscani eds.), Birkhauser, pages 297-336, 2010.

[3] José A. Carrillo, Young-Pil Choi, Eitan Tadmor and Changhui Tan. Critical thresholds in 1D Euler equations with non-local forces. Math. Models Methods Appl. Sci., 26(1):185-206, 2016.

[4] Felipe Cucker and Steve Smale. Emergent behavior in flocks. IEEE Trans. Automat. Control, 52(5):852$862,2007$.

[5] Felipe Cucker and Steve Smale. On the mathematics of emergence. Jpn. J. Math., 2(1):197-227, 2007. 
[6] Raphaël Danchin, Piotr B. Mucha, Jan Peszek, and Bartosz Wróblewski. Regular solutions to the fractional euler alignment system in the Besov spaces framework. arXiv:1804.07611, 2018.

[7] Tam Do, Alexander Kiselev, Lenya Ryzhik, and Changhui Tan. Global regularity for the fractional Euler alignment system. Archive for Rational Mechanics and Analysis, 228(1):1-37, 2018.

[8] Alessio Figalli and Moon-Jin Kang. A rigorous derivation from the kinetic Cucker-Smale model to the pressureless Euler system with nonlocal alignment. arXiv:1702.08087v1, 2017.

[9] Seung-Yeal Ha, Taeyoung Ha, and Jong-Ho Kim. Emergent behavior of a Cucker-Smale type particle model with nonlinear velocity couplings. IEEE Trans. on Automatic Control, 55(7), 2010.

[10] S.-Y. Ha, J. Kim, J. Park and X. Zhang, Uniform stability and mean-field limit for the augmented Kuramoto model. NHM 13(2): 297-322, 2018.

[11] Seung-Yeal Ha and Jian-Guo Liu. A simple proof of the Cucker-Smale flocking dynamics and mean-field limit. Commun. Math. Sci., 7(2):297-325, 2009.

[12] Seung-Yeal Ha and Eitan Tadmor. From particle to kinetic and hydrodynamic descriptions of flocking. Kinet. Relat. Models, 1(3):415-435, 2008.

[13] J. Haskovec. Flocking dynamics and mean-field limit in the Cucker-Smale type model with topological interactions. Phys. D, 261(15):42-51, 2013.

[14] Siming He and Eitan Tadmor. Global regularity of two-dimensional flocking hydrodynamics. Comptes rendus - Mathematique Ser. I, 355:795-805, 2017.

[15] Chunyin Jin. Flocking of the Motsch-Tadmor model with a cut-off interaction function. J Stat Phys, 171:345-360, 2018.

[16] Sebastien Motsch and Eitan Tadmor. A new model for self-organized dynamics and its flocking behavior Journal of Statistical Physics, 144(5): 932-947, 2011.

[17] Sebastien Motsch and Eitan Tadmor. Heterophilious dynamics enhances consensus. SIAM Rev., 56(4):577-621, 2014.

[18] Piotr B. Mucha and Jan Peszek. The Cucker-Smale equation: singular communication weight, measurevalued solutions and weak-atomic uniqueness. Arch. Rational Mech. Anal., 227:273-308, 2018.

[19] J. Peszek. Existence of piecewise weak solutions of a discrete Cucker-Smale's flocking model with a singular communication weight. J. Differential Equations, 257:2900-2925, 2014.

[20] Jan Peszek. Discrete Cucker-Smale flocking model with a weakly singular weight. SIAM J. Math. Anal., 47(5):3671-3686, 2015.

[21] David Poyato and Juan Soler. Euler-type equations and commutators in singular and hyperbolic limits of kinetic Cucker-Smale models. Math. Models Methods Appl. Sci., 27(6):1089-1152, 2017.

[22] R. Shvydkoy. Global existence and stability of nearly aligned flocks. arXiv:1802.08926, 2018.

[23] Roman Shvydkoy and Eitan Tadmor. Eulerian dynamics with a commutator forcing. Transactions of Mathematics and Its Applications, 1(1):tnx001, 2017.

[24] Roman Shvydkoy and Eitan Tadmor. Eulerian dynamics with a commutator forcing II: Flocking. Discrete Contin. Dyn. Syst., 37(11):5503-5520, 2017.

[25] Roman Shvydkoy and Eitan Tadmor. Eulerian dynamics with a commutator forcing III: Fractional diffusion of order $0<\alpha<1$. Physica D, 376-377 (2018) 131-137.

[26] Roman Shvydkoy and Eitan Tadmor. Topological models for emergent dynamics with short-range interactions. arXiv:1806.01371

[27] Eitan Tadmor and Changhui Tan. Critical thresholds in flocking hydrodynamics with non-local alignment. Philos. Trans. R. Soc. Lond. Ser. A Math. Phys. Eng. Sci., 372(2028):20130401, 22, 2014.

[28] Changhui Tan. Finite time blow up for a fluid mechanics model with nonlocal velocity. ArXiv, 2017. 
Center for Scientific Computation and Mathematical Modeling (CSCAMM)

University of Maryland, College Park MD 20742

E-mail address: javierm1@cscamm.umd.edu

Center for Scientific Computation and Mathematical Modeling (CSCAMM)

University of Maryland, College Park MD 20742

E-mail address: jpeszek@cscamm.umd.edu

Department of Mathematics

Center for Scientific Computation and Mathematical Modeling (CSCAMM)

AND

Institute for Physical Sciences \& Technology (IPST)

University of Maryland, College PARK MD 20742

E-mail address: tadmor@cscamm.umd.edu 